\title{
Linfoma primario de pulmón. Serie de casos
}

\author{
JULIO C. VILLARROEL S.*, MÁXIMO ROMANCZUK*, \\ ESTEBAN J. WAINSTEIN*, MARCOS LAS HERAS*, \\ EDUARDO L. DE VITO*, GRACIELA SVETLIZA* y JUAN A. PRECERUTTI*
}

\section{Primary pulmonary lymphoma. A case serie}

Primary Pulmonary Lymphomas (LPP) are infrequent and their clinical manifestations and images are usually nonspecific. Diagnostic delay may be important. The objective of this study was to analyze the LLP in our institution. Between 2003 and 2013, over 1,892 lymphomas were analyzed in our institution. Only 4 of them (0.21\% incidence) were detected as LPP: Non Hodgkin's Lymphoma (n $=2)$, Hodgkin's Lymphoma $(n=1)$, and Intravascular Pulmonary Lymphoma $(n=1)$. Clinical manifestations of the 4 cases presented were unspecific: 1) pulmonary mass and pleural effusion; 2) consolidation with air bronchogram and cavitations; 3) normal images and 4) pulmonary mass. Given these clinical settings, 4 diagnostic methods were used: 1) Computed Tomography-Guided Puncture, 2) Video-Assisted Thoracoscopic Surgery (VATS); 3) VATS guided by positron emission tomography (PET) and 4) thoracotomy. Hence, diagnosis was successfully made between 45 to 90 days from the initial consultation. This report confirms the low incidence of LPP, and its unspecific clinical and radiographic manifestations that may cause delay in diagnosis. PET can contribute to improve diagnostic performance, especially in patients without apparent lung involvement.

Key words: Lymphoma, Hodgkin 's, non Hodgkin's, primary pulmonary lymphoma, intravascular lymphoma, positron emission tomography.

\section{Resumen}

Los linfomas primarios de pulmón (LPP) son infrecuentes. Sus manifestaciones clínicas y las imágenes son inespecificas. El retraso diagnóstico puede ser considerable. Objetivo: Analizar los LPP durante el periodo 2003-2013 en nuestra institución. Sobre 1892 linfomas, 4 fueron LPP (0,21\%): 1) linfoma no Hodgkin $(n=2)$; 2) linfoma Hodgkin $(n=1)$; 3) linfoma intravascular pulmonar $(n=1)$. Las manifestaciones clínicas y radiológicas fueron inespecificas (masa pulmonar y derrame pleural, consolidación con broncograma aéreo y cavitación o bien ausencia de lesiones). Los métodos diagnósticos fueron: 1) punción guiada bajo TAC; 2) videotoracoscopía (VATS) y 3) VATS orientada por PET (tomografía por emisión de positrones) y 4) toracotomía. El tiempo entre la consulta inicial hasta el diagnóstico fue de 45 a 90 días. Este reporte confirma la baja incidencia de LPP, y sus manifestaciones clínicas y radiologías poco específicas. Esto puede contribuir a las demoras en el diagnóstico. El PET puede mejorar el rendimiento diagnóstico, en especial en ausencia de compromiso pulmonar radiológico evidente.

Palabras clave: Linfoma, Hodgkin, no Hodgkin, linfoma pulmonar primario, linfoma intravascular, tomografía por emisión de positrones.

\section{Introducción}

Los linfomas primarios de pulmón (LPP) son tumores confinados al pulmón, sin afección de ganglios mediastinales y sin evidencia de extensión extratorácica durante los tres meses posteriores al diagnóstico ${ }^{1-5}$. Son considerados infrecuentes, constituyen el $0,3 \%$ de todos los tumores malignos de pulmón ${ }^{1-4}$ y el $0,5 \%$ de todos los linfomas ${ }^{3-6}$. Sus manifestaciones clínicas consisten en síntomas respiratorios (tos y disnea), y generales (astenia, pérdida de peso, etc.), que suelen adjudicarse a otras enfermedades más frecuentes ${ }^{4-7}$. Asimismo, las manifestaciones

* Clínica Médica Sección Neumonología, Hospital Italiano de Buenos Aires, Argentina. 
radiológicas y tomográficas pueden ser interpretadas como procesos infecciosos o tumorales más frecuentes. Debido a esto, se ha descripto un retraso de 5 meses a 8 años para llegar al diagnóstico ${ }^{2,6,8,9}$.

El objetivo de este estudio fue analizar los LPP diagnosticados durante el período 2003-2013. Se discuten las imágenes radiológicas pulmonares de presentación, los métodos diagnósticos empleados y se analizan las posibles demoras en el tratamiento.

\section{Caso clínico 1}

Paciente de género femenino de 76 años, consultó por disnea progresiva con CF III, astenia y pérdida de peso de 7 a $8 \mathrm{~kg}$ en los últimos 6 meses. Antecedentes personales: hipercolesterolemia. Examen físico: ausencia de murmullo pulmonar en todo el campo pulmonar derecho y matidez, saturación de $\mathrm{O}_{2} 92 \%$. Laboratorio: Hemoglobina (Hb) 10,2 g/dL, Hematocrito (Hto) $30 \%$, Leucocitos (GB) 6.190 células $/ \mathrm{mm}^{3}(88 \%$ neutrófilos), Velocidad de eritrosedimentacion (VSG) $56 \mathrm{~mm}$. Radiografía de tórax: opacificación del campo pulmonar derecho compatible con derrame pleural. Se realizó una punción del derrame pleural que fue compatible con un exudado no complicado. Comenzó tratamiento con ampicilina-sulbactam EV. Durante su hospitalización presentó un episodio de disnea súbita secundaria a un tromboembolismo pulmonar a nivel del tronco de la arteria pulmonar izquierda. Una tomografía axial computada (TAC) de tórax mostró derrame pleural derecho leve y una masa en lóbulo inferior derecho con broncograma aéreo. Se interpretó como neumonía con derrame pleural y tromboembolismo pulmonar. Se continuó con el mismo esquema antibiótico y se comenzó anticoagulación. Al $7^{\mathrm{mo}}$ día fue dada de alta. A los 30 días consultó al Servicio de Neumonología. Se encontraba afebril, persistía la astenia. Una nueva TAC de tórax evidenció la persistencia de la masa pulmonar con escaso derrame pleural, sin adenopatías mediastinales (Figura 1). Se decidió realizar una punción pulmonar guiada bajo TAC, previa suspensión de la anticoagulación. La punción mostró un proceso linfoproliferativo compatible con Linfoma No Hodgkin (LNH) folicular, de células B de bajo grado, CD 20 y BCL2 positivo. El PET mostró una masa hipercaptante parahiliar derecha (intensidad de captación: standarized uptake value, SUV = 23,2) un foco metabólico en colon transverso (pólipo colónico por fibrocolonoscopía). Comenzó quimioterapia con
CHOP: ciclofosfamida, doxorrubicina, vincristina y prednisona), más el anticuerpo monoclonal rituximab (Rituxan). Este regimen es conocido como R-CHOP. Actualmente (6 meses después) se encuentra en seguimiento por el Servicio de Oncología con buena evolución.

\section{Caso clínico 2}

Paciente de género masculino de 37 años, consultó por tos seca de 2 meses de evolución. Antecedentes personales: Trabaja en el área de salud como administrativo y estuvo en contacto con un compañero con TBC. Examen físico: disminución del murmullo pulmonar en hemitórax superior izquierdo. Laboratorio: $\mathrm{Hb} 16 \mathrm{~g} / \mathrm{dL}$, Hto 47,6\% GB 7.510 (61,1\% neutrófilos) VSG 33 $\mathrm{mm}$. Radiografía de tórax: imagen cavitada en el segmento apical del LSI de paredes gruesas, sin nivel. TAC de tórax: Consolidación a nivel del lóbulo superior izquierdo con signos de broncograma aéreo y sectores cavitados que podría corresponder a un proceso infeccioso (Figura 2). Presenta amplia superficie de contacto con la pleura. Baciloscopía de esputo y PPD negativos, cultivo de lavado broncoalveolar negativo, biopsia transbronquial con proceso inflamatorio inespecífico crónico. Se efectuó una punción guiada bajo TAC que evidenció una imagen compatible con proceso inflamatorio abscedado. El paciente recibió tratamiento con antibióticos por 14 días y posteriormente antifímicos (drogas anti-TBC) por 60 días, sin mejoría de los síntomas. Se realizó lobectomía quirúrgica cuyo resultado fue un Linfoma Hodgkin (celularidad mixta) de pulmón con implantes en pleura, CD 20 positivo. Recibió tratamiento con quimioterapia (R-CHOP). A los 2 años se hallaba en remisión.

\section{Caso clínico 3}

Paciente de género masculino de 71 años. Consultó por hiporexia y pérdida de peso de $9 \mathrm{~kg}$ en los últimos 2 meses. Antecedentes personales: hiperuricemia. Examen físico: sin datos relevantes, $\mathrm{SatO}_{2}$ 96\%. Laboratorio: $\mathrm{Hb} 12,2$ g/dL, Hto $37,2 \%$, GB 4.100 (50\% neutrófilos), Plaquetas $80.000 / \mathrm{mm}^{3}$, VSG $20 \mathrm{~mm}$, lactato deshidrogenasa (LDH): $2670 \mathrm{UI} / \mathrm{L}$. Radiografía de tórax: normal. TAC de tórax y abdomen, leve esplenomegalia. Debido a que la pérdida de peso no tenía causa evidente se efectuó PET-TC (Figura 3), que mostró aumento difuso del metabolismo a nivel de ambos parénquimas pulmonares (SUV 4,5), poco aumento del metabolismo a nivel de médula ósea 


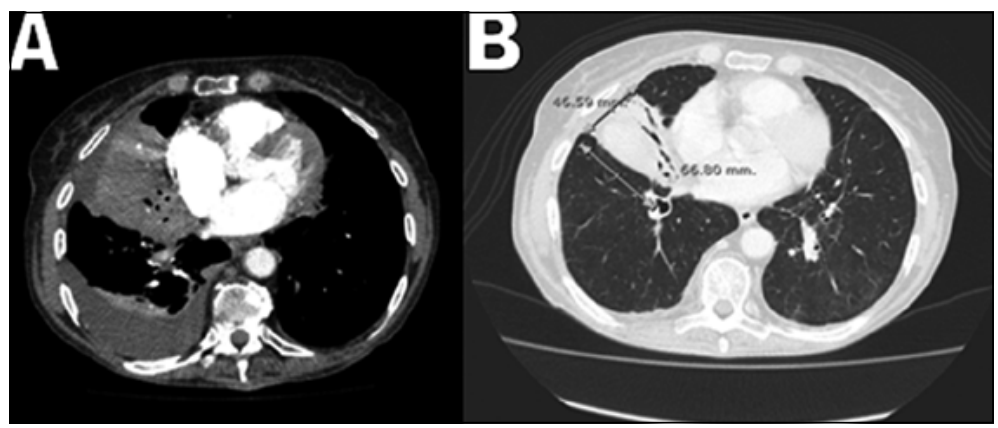

Figura 1. Caso 1, TAC tórax $\mathbf{A} y$ B: se observa una masa que se extiende desde el hilio pulmonar derecho hacia el lóbulo medio y discreta cantidad de derrame pleural derecho. Las medidas de ésta se señalan en la figura.

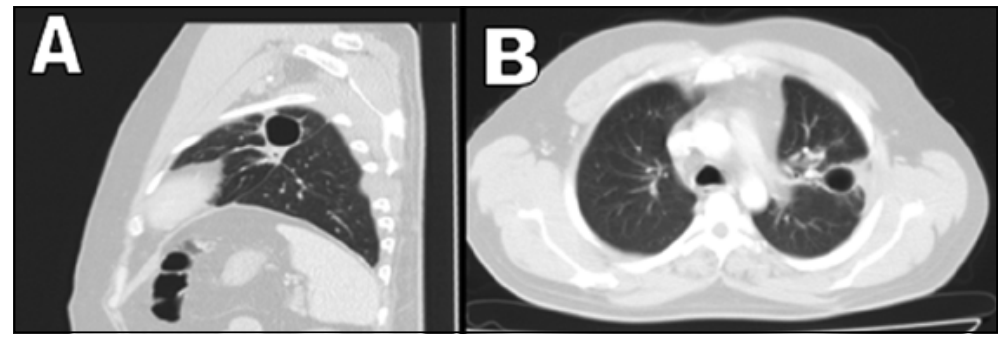

Figura 2. Caso 2, TAC de tórax A y B. Se observa una formación de aspecto quístico con contenido aéreo, de paredes delgadas, asociada a trazos densos y adherencias pleurales a nivel del LSI. Se asocia a dilatación de estructuras bronquiales adyacentes, por tracción.

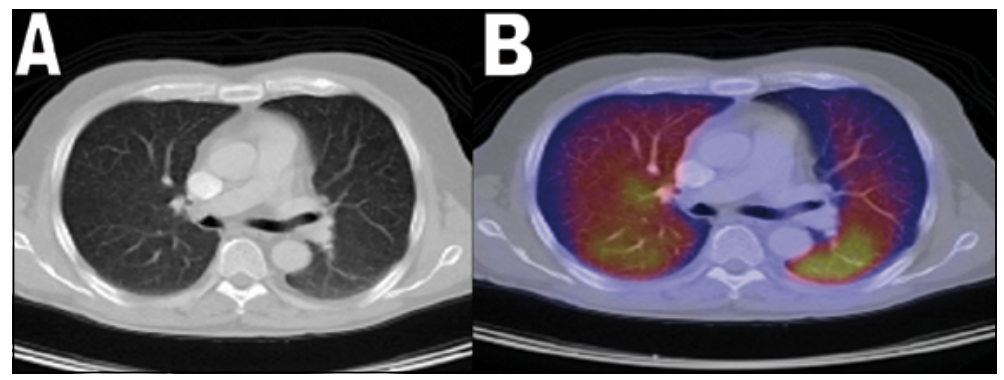

Figura 3. Caso 3, TAC de tórax normal (A), y PET (tomografía por emisión de positrones) con aumento difuso del metabolismo a nivel de ambos parénquimas pulmonares (B).

y no captó en bazo. Los cultivos del BAL fueron negativos, con citometría de flujo macrófagos y células de aspecto normal y leve aumento de linfocitos de células pequeñas. Estudios funcionales respiratorios: $\mathrm{CVF}=3,89 \mathrm{~L}(110 \%)$, $\mathrm{VEF}_{1}=2,80 \mathrm{~L}(104 \%), \mathrm{VEF}_{1} / \mathrm{CVF} \cdot 100=72 \%$, DLCO $=20,10 \mathrm{ml} / \mathrm{min} / \mathrm{mmHg}(90 \%)$, volumen alveolar $(\mathrm{VA})=5,52 \mathrm{~L}(90 \%), \mathrm{DLCO} / \mathrm{VA} 3,64$ $\mathrm{mL} / \mathrm{min} / \mathrm{mmHg} / \mathrm{L}(82 \%)$. Se decidió realizar biopsia pulmonar que informó LNH difuso de células tipo B intravascular (LIV), CD 20 y CD 10 positivo. Comenzó quimioterapia (R-CHOP) con buena evolución a 5 años de seguimiento.

\section{Caso clínico 4}

Paciente de género femenino de 35 años. Tabaquismo de 20 paquetes/año. Consultó por tos y disnea de esfuerzo. Se interpretó como neumonía atípica y recibió tratamiento con antibióticos (claritromicina). Debido a la falta de respuesta terapéutica se efectuó TAC que evidenció una masa ubicada en el lóbulo inferior izquierdo. La fibrobroncoscopía mostró obstrucción de la luz bronquial en el segmento antero-medial. El cepillado y lavado bronquial fueron compatibles con carcinoma poco diferenciado. Se efectuó mediastinoscopía (ganglios normales) y toracotomía y lobectomía inferior izquierda. El informe de anatomía patológica reveló un linfoma $\mathrm{LNH}$ (difuso de células B). Se realizó tratamiento con R-CHOP. Actualmente en seguimiento por hematología ${ }^{4}$.

\section{Discusión}

Sobre un total de 1.892 linfomas registrados en nuestra institución durante el período 2003-2013, se diagnosticaron cuatro LPP $(0,21 \%)$ : dos fueron $\mathrm{LNH}^{4}$, uno LH y el otro LIV. Debido a su rareza, 
las manifestaciones clínicas y las imágenes son inespecíficas, el retraso diagnóstico puede ser considerable ${ }^{9-11}$.

El 50\% de los pacientes suelen ser asintomáticos. Los síntomas respiratorios más frecuentes son tos, disnea, dolor toráxico y hemoptisis. Los síntomas sistémicos como pérdida de peso y fiebre están presentes en el 25\%. En el LIV predominan los síntomas sistémicos y el compromiso pulmonar es raramente diagnosticado en vida aunque se encuentra afección pulmonar en $60 \%$ de las autopsias ${ }^{11}$. Los LIV afectan con mayor frecuencia el sistema nervioso central y la piel. Su incidencia global se estima en un caso por millón de habitantes ${ }^{12}$. El compromiso pulmonar aislado es considerado excepcional. Sin embargo, cuando está presente, el paciente puede presentar hipoxemia. Dos de nuestros pacientes tenían disnea (casos 1 y 4). El paciente con LIV tenía síntomas constitucionales, sin hipoxemia y estudios funcionales normales (caso 3). Los casos 2 y 4 presentaron tos.

En el LNH suelen ser opacidades alveolares únicas (50-90\%) y menores de $5 \mathrm{~cm}$ de diámetro, el $50 \%$ se asocia con broncograma aéreo, pueden ser múltiples $(70-77 \%)$ y bilaterales $(60-70 \%)^{10,11,14}$. Otras manifestaciones menos frecuentes son masa pulmonar y afección pleural $(<10 \%)^{11,15,16}$. En los LH, comprenden en orden de frecuencia nódulos pulmonares múltiples, patrón reticular (afección linfática) y consolidación lobar. El derrame pleural está presente en el $8-16 \%{ }^{16}$. El LIV pulmonar puede presentarse sin anomalías radiológicas o bien puede haber lesiones nodulares y consolidaciones ${ }^{16}$. Se han descrito 2 casos con radiografías de tórax y TAC normales, en los que el PET puso de manifiesto compromiso pulmonar vascular ${ }^{9,11,13}$. Nuestros pacientes se presentaron con derrame pleural masivo y masa pulmonar por debajo (caso 1), con una cavidad de paredes gruesas (caso 2), y sin lesiones radiológicas y tomográficas (caso 3) y con una masa pulmonar asociada a compromiso endrobronquial (caso 4). Estas manifestaciones radiológicas son consideradas infrecuentes en los LPP y suelen evocar diagnósticos de condiciones más usuales (ej. neumonía aguda, cáncer de pulmón, tuberculosis).

El diagnóstico de los LPP se realiza por: biopsia quirúrgica $(70 \%)$, biopsia transbronquial (30$40 \%$ ), punción guiada bajo TAC. Son orientadores de LPP la presencia de linfocitos pequeños en el $\mathrm{BAL}^{11}$, la utilización conjunta de PET-TC en pacientes con sospecha de enfermedades linfoproliferativas ha sido reportada en los últimos años y podría ayudar al diagnóstico. Esta técnica diagnóstica resultó particularmente útil en el caso 3, sin manifestaciones radiológicas y con TAC normal. Debido a la captación difusa del PET, se procedió a efectuar un lavado bronco-alveolar que mostró un predominio de linfocitos pequeños que motivó la biopsia quirúrgica. La utilidad del PET-TC en el LIV ha sido descrita en otros reportes ${ }^{17}$. En general, se acepta que esta técnica de imágenes funcionales híbridas (PET-TC) pueden ser de gran utilidad el demostrar afectación de los órganos a pesar de no observase cambios morfológicos ${ }^{17}$. Se ha descrito la existencia de hasta 6 tumores primarios sincrónicos en un mismo paciente por PET (carcinoma in situ) ${ }^{18}$. Su utilización para el estudio de fiebre de origen prolongado fue de ayuda diagnóstica en el $40 \%$ de los casos. En el caso de los linfomas, hay acuerdo general en que el PET-TC se utiliza para estadificación, seguimiento y evaluación de la respuesta al tratamiento ${ }^{19}$. En nuestros pacientes el PET-TC permitió delimitar la enfermedad al pulmón (caso 1) y poner de manifiesto compromiso pulmonar vascular no evidente por otros métodos (caso 3).

Debido a que las manifestaciones clínicas y las imágenes son inespecíficas, y considerando lo poco frecuentes, el retraso diagnóstico de los LPP puede ser importante. Se estima que entre el momento de las primeras manifestaciones clínicas y la consulta médica transcurrieron entre 2 y 6 meses, mientras que desde el momento de la consulta inicial hasta el diagnóstico de LPP el tiempo transcurrido fue de 45 a 90 días. Se ha descripto que el $45 \%$ de los considerados LPP al momento de la cirugía tenían afección mediastinal ${ }^{1,15}$. Nuestros pacientes no tenían evidencias de compromiso mediastinal (por cirugía o por PET).

Este reporte confirma la baja incidencia de LPP, y sus manifestaciones clínicas y radiologías poco específicas. Esto puede contribuir a las demoras en el diagnóstico. Si bien no es infrecuente el diagnóstico mediante anatomía patológica o autopsias $^{1,15}$, estos casos fueron diagnosticados en vida. Por todo esto, los LPP siguen siendo un desafío diagnóstico. En algunos casos el PET puede contribuir, a mejorar rendimiento diagnóstico, en especial en pacientes sin compromiso pulmonar manifiesto en las imágenes y probablemente pueda contribuir a mejorar el pronóstico si se detecta en etapa temprana ${ }^{16}$.

\section{Bibliografía}

1.- CADRANEL J, WISLEZ M, ANTOINE M. Primary pulmonary lymphoma. Eur Respir J; 2002; 20: 750-62.

2.- MARTÍNEZ RIVERA C, BONNIN VILAPLANA M, SIMÓN ADIEGO C, PALACÍN FORGUÉ A, PUIG 
ZUZAA J, SAMPABLO LAURO I. Masa cavitada como forma de presentación de un linfoma pulmonar primario. Arch Bronconeumol 2004; 40: 94-6.

3.- ZUCKERMAN D. The intravascular lymphoma: the oncologist great imitator. Oncologist 2006; 11: 496-502.

4.- DA LOZZO A G, MAZZINI F, DALURZO M, SMITH D, BEVERAGGI E. Linfoma pulmonar primario, presentación de un caso. 78vo Congreso Argentino de Cirugía, 2007. Consultado el 13/01/2014: http:// www.hospitalitaliano.org.ar/personas/conf_contenido/.

5.- SANCHO CHUST J N, CHINER VIVES E, CAMARA-

SA ESCRIG A. Linfoma pulmonar primario: una rara causa de masa pulmonar. Anales de Medicina Interna 2008; 25: 347-80.

6.- TAKAHASHI R, NISHIKAWA M, NOMI F, KUSANO N, KAKEMIZU N, ISHIGATSUBO Y. Case of intravascular lymphoma diagnosed by transbronchial lung biopsy with transient spontaneous remission. Nihon Kikyuki Gakkai Zasshi 2010; 48: 825-30.

7.- LUCH GARCÍA R, BRIONES GÓMEZ A, MONZO CASTELLANO E, SÁNCHEZ TORIL F, LÓPEZ A, BROTONS B. Primary Pulmonary Hodgkin's lymphoma. Can Respir J 2010; 17: 106-8.

8.- QIN L, LIU H R, FENQ R E, LIU T, LI J, LU W, QIN M W. Non-Hodgkin's lymphoma with diffuse ground glass opacity on chest CT: a report of 6 cases. Zhonghua Yi Xue Za Zhi 2010; 90: 3283-6.

9.- TOMIHARU N, KIKUO I, KOJI M, SOICHIRO K, HIDEO K, AYAKO K, et al. Pulmonary intravascular lymphoma diagnoseed by 18 -flurodeoxyglucosa positron emission tomography-guided transbronchial lung biopsy in a man with long term survival: a case report. J Medical Case Reports 2011; 5: 295-30.

10.- JUANG H, LU P X. Classical Hodking lymphoma infiltrated both lungs. Quant Imaging Med Surg 2012; 2:
288-90.

11.- PARISSIS H. Forty Years literature review of primary lung lymphoma. J Cardiothoracic Surgery 2011; 6: 1-23.

12.- TAPIA O, JIMÉNEZ P. Linfoma intravascular de células B grandes: hallazgos clínicos y morfológicos en un caso con desenlace fatal. Rev Med Chile 2012;140: 225-30.

13.- YAMASHITA H, SUZUKI A, TAKAHASHI Y, KUBOTA K, KANO T, MIMORI A. Intravascular large B cell lymphoma with diffuse FDG uptake in the lung by FDG-PET/CT without chest CT findings. Ann Nuclear Med 2012; 26: 515-21.

14.- AZIZ O, FOUAD A, LHOU F A, MUSTAPHA I R. Lymhoma non Hodgkin en pulmonaire primitif a propos d'un cas. Pan African Medical Journal 2012; 11: 56.

15.- EZZINE BACCARI S, BACHA D, BOUZAIDI K, GHRAIRI H, SASSI S. Hodgking Lymphoma present with Exclusive Pulmonary Involvement. Tunis Med 2012; 90: 833-4.

16.- JOSETTE W, DAINA V, ANJANA Y, KIRTEE R. Lymphoproliferative Neoplasms of the Lung: A Review. Arch Pathol Lab Med 2013; 137: 382-91.

17.- KOHAN A A, PAGANINI L, BIEDAK P, ARMA J I, DARLUZO M C, GARCÍA MONACO R D. Pulmonary Intravascular Limphoma Detected by FDG PET-CT: A case report. Rev Esp Med Nucl Imagen Mol 2013; 32: 318-5.

18.- ADRIAENSEN M, SCHIJF L, DEHAAS M, HUIJBREGTS J, BAARSLAG H J, STAAKS G, et al. Six synchronous primary neoplasms detected by FDG-PET/ CT. Eur J Nucl Med Mol Imaging 2008; 35: 1931.

19.- MALDONADO A, GONZÁLEZ ALENDA A, ALONSO B M, SIERRA B J. Utilidad de la tomografía por emisión de positrones-tomografía computarizada (PET-TC) en neumología. Arch Bronconeumol 2007; 43: 562-72.
Correspondencia a:

Dr. Julio César Villarroel Saavedra

Clínica Médica, Sección Neumonología

Hospital Italiano de Buenos Aires

Gascón 450, Buenos Aires, C.F., Argentina.

Email: julio.villarroel@hospitalitaliano.org.ar 